\title{
Recomendações para o design de textos e hiperlinks em aplicativos móveis culturais
}

\author{
Text and hyperlink recommendations for \\ designing mobile culturais guides
}

interfaces móveis,

Este artigo descreve recomendações para o uso de texto e hiperlinks em interfaces multimídia,

testes com usuários

\section{Heloisa Candello}

mobile Interfaces, multimedia, user tests móveis. Estas recomendações fazem parte de um conjunto de recomendações de como apresentar conteúdos multimídia culturais para serem mostrados em telefones celulares acessados em espaços abertos por seus usuários. O estudo comparou dois modos de mostrar texto na tela de um aparato móvel: o primeiro em texto corrido e o segundo com hiperlinks. No total, 32 participantes avaliaram o sistema. Como resultado, o estudo sugere que o design de telas contendo texto corrido e hiperlinks com informações culturais devem considerar a idade, a nacionalidade, familiaridade tecnológica dos usuários, bem como a compreensão do conteúdo na tela, a motivação em aprender novos conceitos e as expectativas dos usuários.

This paper aims to describe recommendations for text and hyperlink use in mobile interfaces. These recommendations belong to a cluster of recommendations of how to present multimedia content in mobile cultural guides to be accessed in open air spaces. The study compared two modes to present text on a mobile screen: first a full body text and second a text with hyperlinks. Overall, third-two participants evaluated those modes. As a result, the study suggests that designers should take in consideration some factors while designing layouts with text, such as: age, nationality, technology familiarity, as well comprehension of information on the screen, motivation to learn new concepts and user expectations.

\section{Introdução}

Este trabalho é parte integrante de uma pesquisa maior (CANDELLO, 2012) relacionada ao design de dispositivos móveis para serem acessados em espaços abertos. O escopo deste trabalho é restrito a atividade de uso de telefones touch screen para acessar conteúdo multimídia de pontos culturais e promover recomendações de design para desenvolver estes tipos de aplicativos móveis. Neste artigo, são enfatizadas somente as recomendações para o uso de texto e 
hiperlinks em interfaces de dispositivos móveis. Essas recomendações são resultados de um estudo que foi dividido em quatro etapas. Um primeiro estudo de campo foi realizado para desvendar como os guias turísticos passavam informações para os visitantes de um espaço cultural (CANDELLO, 2008). Segundo, como as informações culturais eram consultadas em guias de papel (CANDELLO 2011a, 2011b). Terceiro, como as mesmas informações foram acessadas através de um dispositivo móvel (CANDELLO, 2011c). Estes três estudos geraram requisitos de design para o desenvolvimento de um protótipo funcional - um guia cultural móvel - e dois modos de apresentar textos em interfaces móveis foram testados por potenciais usuários, o que consistiu no quarto estudo.

\section{Projetos correlatos}

Princípios para o design de interfaces móveis são essenciais para guiar tanto designers quanto profissionais da área cultural. Alguns projetos se concentram em diretrizes para o desenvolvimentos de aplicativos móveis culturais. Por exemplo, o modelo criado para assistir na aplicação de diferentes cenários para guias baseados em mapas desenvolvido por Grun (2005). Além deste, Savio and Braiterman (2007) apresentam heurísticas para interação móvel considerando as limitações dos usuários, contexto de uso e do aparato móvel. No mesmo sentido, Paay and Kjeldskov (2007) criaram um método para prover conhecimento aos designers de aplicativos móveis sobre identificação de elementos presentes no contexto do usuário, e esta informação pode ser indexada na interface do aplicativo. No contexto de aprendizagem usando apps móveis, Grasso and Roselli (2005) desenvolveram guidelines para criar conteúdos e cursos em telefones celulares. Mais recentemente, Eliasson e Ramberg (2012) apresentam seis guidelines para o design de atividades de aprendizagem nos telefones móveis considerando a localização dos estudantes e o contexto destas atividades, onde os telefones celulares ajudam ao invés de distrair estudantes do conteúdo e do contexto relevante para os objetivos da aprendizagem. Sá \& Churchill (2013) oferecem aos designers de guias móveis que utilizam a tecnologia de realidade aumentada recomendações de quando usar no processo de design diferentes níveis de fidelidade de prototipagem.

Em relação ao uso de texto e hiperlinks in interfaces móveis, Schilit et al (2002) afirmaram que o uso de hiperlinks e separar o texto de páginas WEB em diferentes interfaces é mais prático para navegação e a manipulação de conteúdo. Porém. Desconsideravam ação do usuário, que naquele contexto era mais relevante do que nos dias de hoje, pois eram telefones com teclado e que não possuíam telas de toque (touchscreen). Sato et al (2007) propõem um modelo que transforma a interface do usuário em uma interface customizável usando hiperlinks para acessar aplicativos, porém não explora hiperlinks no corpo de 
texto somente na organização das informações na interface. Hyeju et al (2010) mostra as vantagens de se usar hipertexto combinando com técnicas de busca. No modelo proposto, o sistema identifica as possíveis palavras em um texto que os usuários desejam saber mais e transforma em hipertexto, facilitando a busca e minimizando os cliques.

Todas estas perspectivas são importantes, entretanto, há uma escassez de estudos que investigam como as pessoas interagem com as representações de informações nas telas dos telefones celulares, em diferentes contextos.

\section{Guia móvel dos monumentos de Brighton - UK}

O estudo foi realizado na cidade de Brighton \& Hove, no Reino Unido em 2010. Participantes utilizaram um protótipo, que foi desenvolvido baseado em estudos anteriores (CANDELLO, 2008, CANDELLO, 2011a, CANDELLO, 2011b). O texto apresentado no protótipo foi corrigido por especialistas em patrimônios culturais, para evitar qualquer uso inadequado de informação. O protótipo apresenta dois modos de apresentação de texto. Um texto com hiperlinks e outro com texto corrido. A tipografia de ambos os textos estava em 14pt em Arial. O texto na cor hexadecimal \#1A1A1A e o fundo de tela branco. As perguntas que guiaram esta investigação foram:

- A tipografia, cores e fundo de tela utilizados são adequados para a leitura em espaços abertos?

- O perfil dos usuários afeta o acesso aos hiperlinks e a leitura do texto?
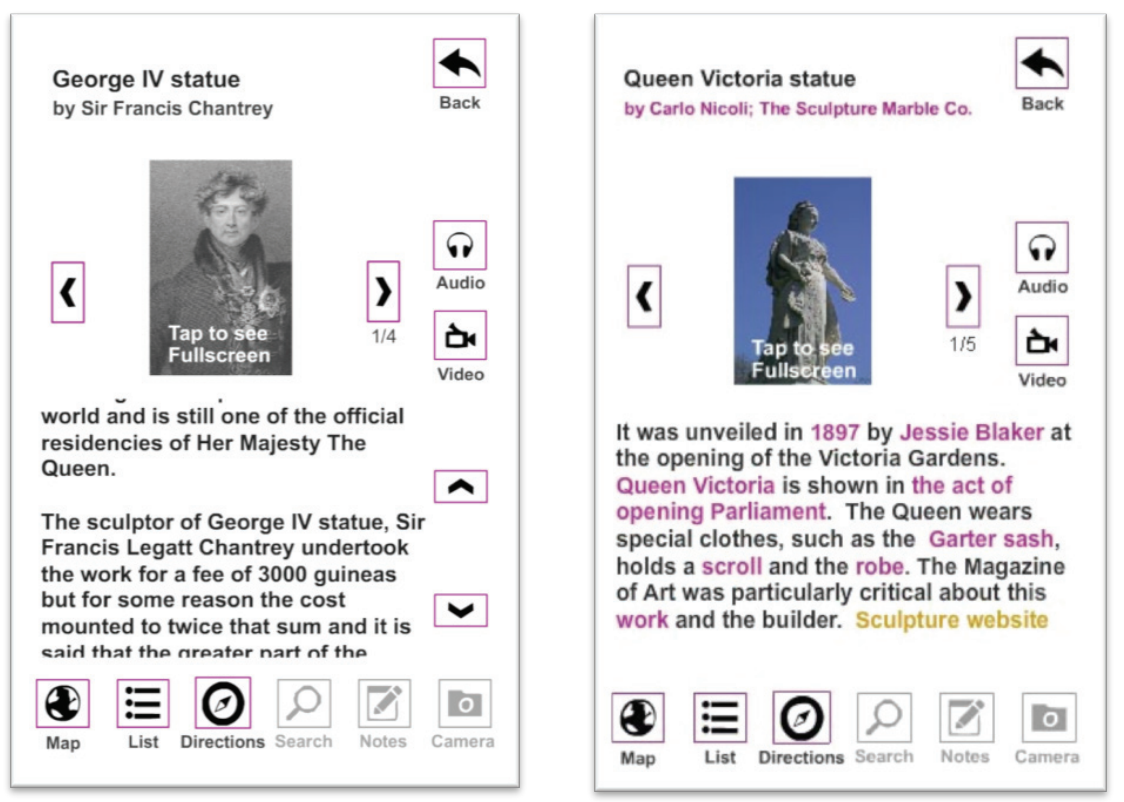

Figura 1 Modos de apresentação de texto no protótipo 


\subsection{Participantes do experimento}

Trinta e dois participantes fizeram o estudo. A idade dos participantes era entre 18 e 71 anos. Entretanto, a maioria dos participantes tinha entre 23 e 29 anos. A maioria dos participantes tinha touch-screen telefones ou já tinha usado este tipo de dispositivo antes. De acordo com os dados, $50 \%$ dos participantes usavam telefones touch-screen todos os dias, $37,5 \%$ não frequentemente e $12,5 \%$ nunca tinham utilizado este tipo de telefone celular anteriormente. Participantes que usavam este tipo de telefone todos os dias eram mais novos do que aqueles que nunca tinham usado ou não usavam frequentemente este tipo de dispositivo. Os dados foram bem variados no que diz respeito à nacionalidade.

Participantes eram de origens variadas: Europa (56\%), América do Sul (25\%) e Asia (19\%). Considerando que $28 \%$ dos participantes eram do Reino Unido, foi relevante ter este diverso grupo de participantes para identificar possíveis diferenças culturais. Igualmente, o número de pessoas que moravam em Brighton foi um fator que poderia ter afetado os resultados. Quando o recrutamento de participantes foi realizado, questionários foram utilizados para balancear os convites para o estudo em relação a este tópico. Sessenta por cento dos participantes moravam em Brighton por mais de três meses, incluindo os participantes britânicos. Quarenta por cento eram visitantes ou estavam morando em Brighton por menos de três meses. O nível de inglês dos participantes que fizeram o estudo era alto. Vinte por cento consideraram-se pertencentes ao nível intermediário, e o restante eram nativos ou pertencentes ao nível avançado de inglês. Mesmo com um pequeno número de participantes com o nível intermediário de inglês, foi possível identificar algumas diferenças. Diferentes razões trouxeram os participantes para fazer o estudo. Alguns eram muito interessados em tecnologia, outros tinham interesse no conteúdo relativo ao patrimônio cultural. Participantes de diferentes áreas ofereceram diferentes perspectivas durante o estudo. Por exemplo, usuários envolvidos com a área artística (ilustração, música, mídia e patrimônio cultural) exploraram mais o aplicativo e acessaram mais funções. Outros da área de Interação homem computador e Ciências da Computação prestaram mais atenção na funcionalidade e coerência das ações no aplicativo.

O interesse dos participantes no conteúdo, como sua profissão, foi também um dado coletado relevante. Setenta por cento dos participantes estavam interessados no tema do aplicativo. O número de participantes que tinham usado previamente áudio e video guides confirmava o interesse dos mesmos no conteúdo do aplicativo. Oitenta por cento dos participantes tinham usado audio guides anteriormente em museus e galerias de arte (72\%) e em cidades ( $8 \%)$. Isto mostra que eles queriam saber mais sobre as exposições que eles frequentaram. Por outro lado, somente $18 \%$ dos participantes tinham experiência com video guides. Este resultado, abriu oportunidades 
para obter opiniões sem julgamentos baseados em experiências prévias.

A última informação requisitada no questionário foi a disponibilidade deles para a realização do estudo. Setenta e dois por cento dos participantes declararam que eles tinham no mínimo uma hora e no máximo duas horas disponível e quinze por cento entre quinze minutos e meia hora para fazer o tour. O último grupo, em sua maioria, não era familiar com dispositivos touch-screen. Além disso, dois deste participantes fizeram o tour durante a noite depois de passearem o dia todo na cidade. Eles tinham que retornar para Londres depois do tour e afirmaram que estavam cansados. Em geral, usuários esperavam gastar uma a duas horas fazendo o tour usando o telefone celular.

\subsection{Métodos}

Os participantes seguiram um pequeno tour utilizando o telefone celular. O aplicativo do guia estava disponível via mobile banda larga no browser. $\mathrm{O}$ aplicativo tinha um mapa com os pontos de interesse, porém somente dois pontos tinham as informações completas: Queen Victoria e George IV. A pesquisadora pediu aos participantes para saírem da sua localização e irem até um dos monumentos. Dezoito participantes começaram o tour vendo a Queen Victoria estátua e o restante George IV estátua para balancear qualquer efeito de ordem de acesso ao conteúdo. (TULLIS\&ALBERT, 2008).

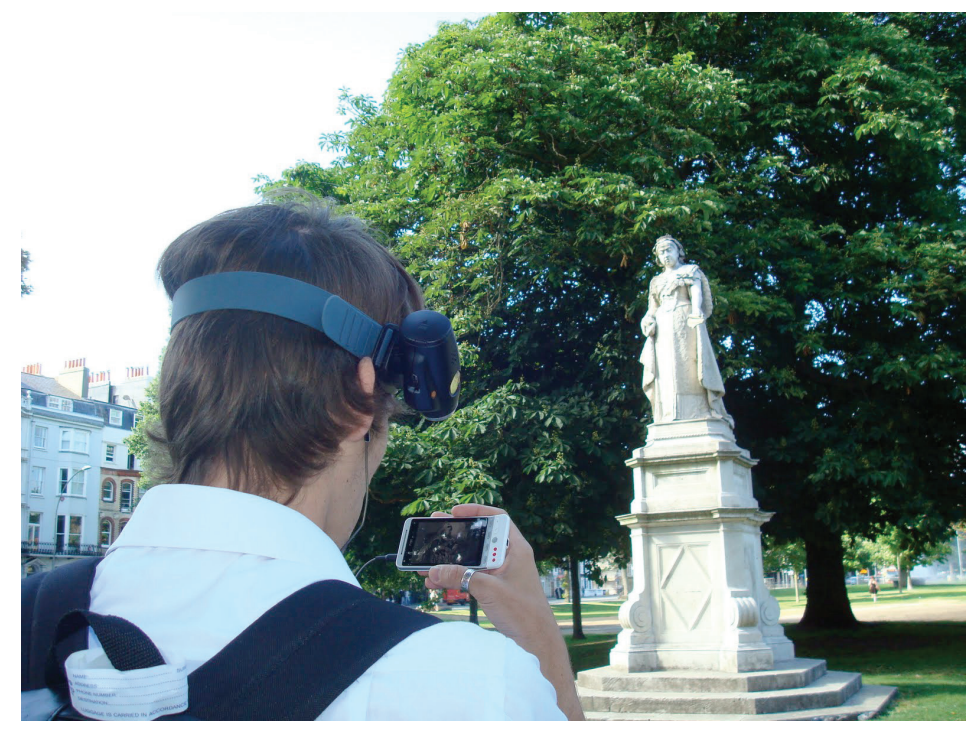

Figura 2 Foto participante fazendo o tour

Na primeira parte do tour, participantes estavam livres para acessar qualquer conteúdo do guia. Na segunda parte do tour, a pesquisadora motivava os mesmos, se necessário, para olhar para informações que 
eles não haviam acessado anteriormente. A pesquisadora marcou a sequencia de acesso do conteúdo multimídia em um formulário durante o estudo. A pesquisadora acompanhou os participantes e pediu que usassem a técnica Think Aloud (Love 2005:64; Preece 2007). Quando os participantes ficavam por algum tempo em silêncio, a pesquisadora gentilmente avisava que ela estava interessada em saber o que eles estavam pensando. Questionários foram preenchidos antes e depois dos tours. Antes do tour, informações demográficas e um formulário de consentimento de gravação para o estudo foram preenchidos. Depois do tour, participantes responderam questões relacionadas à interface do aplicativo e suas preferencias de apresentação e mídia. Anotações foram feitas para garantir que informações relevantes não fossem perdidas, caso a gravação da voz e do vídeo não funcionassem.

\subsection{Análise dos Dados}

Os dados foram analisados usando métodos estatísticos descritivos e métodos qualitativos. Dados estatísticos foram extraídos de dados coletados de questionários - dados demográficos, escalas semânticas e preferências de design. Tabelas e tabulações cruzadas foram aplicadas para comparar os resultados entre os participantes e o uso do sistema. O restrito número de participantes no estudo não foi suficiente para assegurar a validade da análise estatística. Mesmo assim, este estudo identificou dados qualitativos que podem ser utilizados para o design de aplicativos móveis. Considerando isto, concorda-se com as ideias de Lawnson (2006, p. 64), "nós normalmente medimos e expressamos quantidades através de contagens aplicando um sistema numérico. Isto nos leva a acreditar que todos os números se comportam da mesma maneira e isto não é verdade". O mesmo autor enfatiza que o que os designers realmente precisam é saber o significado por trás dos números ao invés de ter somente precisos métodos para calculá-los.

A análise dos dados foi baseada em transformação de dados. A transformação de dados é a quantificação dos dados qualitativos. (CRESWELL, 2009). Isto envolve criar códigos e temas qualitativos, e depois contar o número de vezes que eles ocorrem nos dados de texto. Usando este método os pesquisadores podem comparar resultados quantitativos com qualitativos.

As transcrições dos vídeos, anotações realizadas durante o estudo de campo e sugestões dadas pelos participantes enquanto eles responderam os questionários foram consideradas.

As principais variáveis independentes consideradas foram: idade, residência, nível de inglês, familiaridade com telas móveis touch, experiência prévia com guias móveis, tempo disponível para fazer o tour e fatores ambientais. Algumas variáveis foram adicionadas durante o processo, como ocupação e interesse por patrimônio cultural. Estes mostraram sua relevância no decorrer do processo 
de análise. Variáveis independentes foram usadas para investigar a interação dos participantes com o aplicativo.

As variáveis dependentes, o que nós objetivamos medir, era primeiramente o uso de recursos multimídia nos telefones celulares em espaços abertos. Para este artigo, um recorte foi realizado, e são mostrados os principais resultados em relação à análise de leitura, e uso de hiperlinks nos telefones celulares. Da mesma forma, algumas variáveis dependentes apareceram durante a análise. Tópicos relevantes foram classificados em grupos de códigos, como por exemplo: uso de recursos multimídia, multitarefas, modos de interação com a tela, interesse no conteúdo, limitações do uso desta tecnologia em espaços abertos e públicos e problemas com a tecnologia. Essas variáveis foram combinadas com as variáveis independentes para responder a proposta da pesquisa.

No processo de análise dos vídeos, uma tabela foi utilizada para identificar quais os recursos participantes acessaram durante o experimento e qual foi a ordem de acesso. Somente o primeiro estágio do experimento, foi levado em consideração para fazer a tabela, pois nesta parte do experimento participantes eram instruídos a escolher os recursos que tinham vontade de acessar. Algumas questões emergiram destes dados coletados, como o números de recursos acessados no primeiro monumento visitado, quais as funções que eles acessaram mais e a relação entre as limitações ambientais às escolhas realizadas.

As transcrições dos videos, o estudo de observação e a codificação dos dados foi feita com a ajuda do software NVivo 8. A análise quantitative foi feita com o software Minitab16.

\subsection{Resultados}

Os principais fatores que afetaram os participantes que fizeram o tour foram incluídos em um modelo de integração, para facilitar o acesso de designers de sistemas similares as recomendações sobre o uso de recursos multimídia. A experiência do usuário de guias móveis, de acordo com este estudo está relacionada com fatores relativos ao conteúdo; contexto; e aparência e experiência (Look and Feel) com o design da interface.

Para o recorte feito para este artigo, o acesso ao texto e hiperlinks, foi classificado como um elemento do design da interface (look and Feel). Os fatores coletados que podem influenciar na experiência do usuário com o uso de texto e hiperlink foram: idade do usuário, nacionalidade, compreensão, familiaridade com a tecnologia, tempo disponível para realizar o tour, preferencia de recurso de uso, motivação e expectativa. 
3.5 Recomendações para o uso de recursos multimídia em apps culturais

Entre as noventa recomendações identificadas no estudo, quatorze são relativas sobre o uso de texto e hiperlinks em guias móveis para espaços abertos. Estas serão descritas aqui. Recomendações foram avaliadas sendo de alta importância, média e importante, por meio da quantidade de vezes que o tópico que gerou a recomendação apareceu no estudo. Recomendações de alta importância são mais confiáveis (SC - strenght of confidence) que as somente importantes, as quais precisam ser mais testadas. Recomendações de alta importância são resultados de mais de 10 vezes $-10^{*}$ de um tópico recorrente; $5^{*}-10^{*}$ são as recomendações de média importância e 1 - $4^{*}$ são denominadas recomendadas. A relação entre o fator que gerou o tópico também foi mapeada.

R1 - O texto deve aparecer só em uma janela evitando o uso de barra de rolagem.

SC: $* * *(3)$

Tópico: Quando o bloco de texto não cabe na mesma tela, o tamanho do tipo é considerado menor que quando todo o bloco de texto cabe na mesma tela.

Relação: Idade x texto

3.6 Recomendações para o uso de textos e hiperlinks em apps culturais

Em geral, participantes acessaram quatro recursos para acessar informações na primeira parte do estudo. Texto (56\%) foi o primeiro recurso escolhido para acessar informação, seguido do acesso ao áudio (28\%). Hiperlinks foram pouco acessados pelos visitantes da América do Sul e Ásia, eles estavam em busca de informações básicas, pois estavam na cidade somente por um dia. Por outro lado, residentes acessaram mais os hiperlinks e exploraram o sistema em busca de novas informações. Segue uma lista de recomendações para o uso de texto e hiperlinks em aplicativos móveis para espaços abertos:

\section{R1 - O texto deve aparecer só em uma tela evitando o uso de barra de rolagem.}

SC: *** (3)

Tópico: Quando o bloco de texto não cabe na mesma tela, o tamanho do tipo é considerado menor que quando todo o bloco de texto cabe na mesma tela.

Relação: Idade x texto

R2 - Use o mesmo modelo de interação do dispositivo no aplicativo. SC: $* * * * * *(6)$

Tópico: Foi difícil seguir o texto do monumento George IV.

Relação: Familiaridade com a tecnologia x texto 
R3 - Disponibilize informações através de áudio e texto.

$\mathrm{SC}: * *(2)$

Tópico: Participantes preferiram ler o texto ao invés de ouvir o áudio em ambientes com barulho.

Relação: Compreensão x ambiente $\mathrm{x}$ texto

R4 - Se o usuário acessar o texto, o arquivo de áudio não deve parar automaticamente, somente no caso de acesso de recursos que tenham som (vídeo).

SC: $* * * * * * * * * *(10)$

Tópico: Participantes apreciaram ver o texto e continuar ouvindo o arquivo de áudio.

Relação: Expectativa x áudio x texto

R5 - Guias culturais móveis devem mostrar mensagens de texto para motivar os usuários a olhar para os monumentos.

$\mathrm{SC}: * * * * * * * * * * * * * * * * * * * * *$ (21)

Tópico: Mensagens motivaram os participantes a olhar para os monumentos ao invés de ficarem olhando para a tela do celular. Relação: Motivação x áudio x texto x Ponto de interesse

R6 - Quando os monumentos são muito altos, informações textuais ou de áudio deveriam indicar que estes monumentos são melhores vistos à distância.

SC: **** (4)

Tópico: As pessoas não conseguiam observar o monumento todo quando estavam muito perto destes.

Relação: Motivação x áudio x texto x Ponto de interesse

R7 - Mude o termo "apreciar (appreciate)" por "ver os detalhes" no texto sobre o monumento.

SC: $* *(2)$

Tópico: Internacionais não seguiram as sugestões para chegarem mais perto do monumento como os nativos e participantes nacionais, pois não compreenderam a palavra.

Relação: Motivação x Nacionalidade x texto

R8 - O texto deve rolar, na barra de rolagem, suavemente e fácil de se controlar.

SC: $* * *(3)$

Tópico: Os participantes não conseguiram rolar a barra de rolagem no ritmo que eles desejaram.

Relação: Expectativa x texto

R9 - Apresente diferente formas de apresentar informações, com mais de 45 anos não são sempre pacientes para aprender novas formas de acessar o conteúdo, ou apresente como acessar e ver outras formas de interação sem ser texto. 
SC: $* * * * * *(6)$

Tópico: Pessoas acima de 45 anos leram o texto primeiramente e mais frequentemente do que acessaram vídeos.

Relação: Idade x texto X Vídeo

R10 - Texto é um modo essencial de mostrar informações em um guia móvel, seguido de áudio.

SC: $* * * * * * * * * * * * * * * * *$ (17)

Tópico: Participantes leram mais os textos do que acessaram vídeos, áudio e viram fotos.

Relação: Preferência x texto

R11 - Use hyperlinks para informação extra.

$\mathrm{SC}: * * *(3)$

Tópico: Referências à diversos pontos de interesse no corpo de texto confundiu os participantes.

Relação: Compreensão x texto x hiperlinks

R12 - Use hiperlinks para facilitar os usuários a lerem o texto em espaços abertos.

SC: $* * * * * * * * * * * * * * * * * * * * * * *$ (19)

Tópico: Participantes afirmaram que eles preferem ter hiperlinks do que barra de rolagem. .

Relação: Preferência x texto x hiperlinks

R13 - Hiperlinks devem estar disponíveis para usuários que possuem alguma familiaridade com o tema e querem saber mais sobre isto.

SC: $* * * * * *(6)$

Tópico: Internacionais que passaram somente um dia em Brighton não acessaram os hiperlinks como os residentes e nativos.

Relação: Tempo disponível x texto x hiperlinks

R14 - Hiperlinks devem estar de acordo com o modelo mental dos usuários (no padrão web), ou adicione um ícone SC: $* * * * * * * * * * * *(11)$

Tópico: Enquanto acessavam o texto, nem sempre os participantes identificaram as palavras coloridas como hiperlinks.

Relação: Familiaridade com a tecnologia $\mathrm{x}$ texto $\mathrm{x}$ hiperlinks

\section{Considerações Finais}

Este artigo descreveu um estudo de campo para identificar recomendações para o uso de recursos multimídia em guias culturais móveis para espaços abertos. As recomendações descritas limitaram-se, neste artigo, ao emprego de hiperlinks e maneiras de mostrar o conteúdo no corpo de texto em telas de telefones celulares. 
No total quatorze recomendações foram exemplificadas, bem como o tópico que deu origem a estas e o nível de confiança que podemos ter na aplicação das mesmas.

As duas perguntas, relativas ao uso de texto e hiperlinks podem ser respondidas:

- A tipografia, cores e fundo de tela utilizados são adequados para a leitura em espaços abertos? Sim, a tipografia utilizada no protótipo foi avaliada como tendo boa legibilidade, bem como o contraste entre fundo e tipografia.

- O perfil dos usuários afeta o acesso aos hiperlinks e a leitura do texto? Sim, fatores como idade, familiaridade com a tecnologia, nacionalidade e tempo disponível para fazer o tour afetam a leitura do texto e as preferências por apresentação do mesmo.

A preferência por hiperlinks foi um tópico que agradou tanto visitantes quanto residentes. Porém, o acesso aos hiperlinks foi feito mais por visitantes e nativos do que por turistas que estavam procurando informações mais básicas e sucintas. Por outro lado, nativos e residentes tinham mais tempo para fazer o tour e já tinham familiaridade com o tópico do tour, o que levou os mesmos a explorar mais o sistema em busca de informações novas. Portanto, aplicativos deste tipo podem ter camadas de informações diferentes que satisfaçam tanto iniciantes no assunto como usuários mais especialistas.

Mais estudos precisam ser feitos em relação à preferência e legibilidade da apresentação do texto em espaços abertos. Neste trabalho, somente um tipo de tipografia, fundo de tela e cor de texto foi testado. O que abre oportunidades para uma maior investigação.

\section{Referências}

CANDELlo, H. (2012). Design for outdoor mobile multimedia: representation, content and interactivity. Tese (Doutorado) - Department of Computer Science and Engineering, University of Brighton, Grã-Bretanha.

CANDello, H. ; PEmberton, L.. (2011). Gathering Requirements To Develop Outdoor Mobile Cultural Guides In The Field. In: WCCA 2011 - World Congress on Communication and Arts, 2011, São Paulo. WCCA 2011 - World Congress on Communication and Arts (2011a).

CAndello, H. \& Pemberton, L. (2008). Modelling Behaviour in Guided Tours to Support the Design of Digital Mobile City Guides. MLearn 2008. Ironbridge Gorge, Shropshire, UK: University of Wolverhampton (2008).

CANDELlO, H. (2011). Métodos para a avaliação de guias culturais móveis para o uso em espaços abertos. In: CIDI $\mid 5^{-}$Congresso Internacional de Design da Informação, 2011, Florianópolis. Anais do $5^{\underline{0}}$ Congresso Internacional de Design da Informação, v. 5 (2011b). 
CANDEllo, H.; PEMBERTON, L. (2011). Analysing the experience of being guided by a mobile guide app. Revista GEMInIS, v. 2, p. 21-52 (2011c).

CRESWELL, J. W. (2009). Research Design: Qualitative, Quantitative, and Mixed Methods Approaches, California: Sage Publications.

ELIASSON, J. ET AL. (2013). Design Guidelines for Location-Based and Contextual Learning Supported by Mobile Devices, International. Journal of Handheld Computing Research (IJHCR) 3 (2012): 2, accessed (September 01, 2013), doi:10.4018/jhcr.2012040102

GRASSO, A. \& ROSELLI, T. (2005). Guidelines for Designing and Developing Contents for Mobile Learning. Proceedings of the IEEE International Workshop on Wireless and Mobile Technologies in Education. IEEE Computer Society. GRÜN, C. (2005). Mobile Tourist Guides: Evaluation of the State of the Art and Development of a Light-weight Framework for Location-based Services. Vienna University of Technology. Linz, Austria: Johannes Kepler Universität Linz.

Hyeju, J., ET AL. (2010). Personal Information Access Using Proactive Search and Mobile Hypertext. Intelligent Systems, IEEE 25(1): 27-36. IEE.

LAwson, в. (2006). How Designers Think, Oxford: Architectural Press.

LOVE, s. (2005). Understanding Mobile Human-Computer Interaction, Oxford: Elsevier.

PAAY, J. \& KJELDSKOV, J. (2005). Understanding and modelling built environments for mobile guide interface design Behaviour and Information Technology, 24, 21-35. PREECE, J. Y. ROGERS \& H. SHARP. (2007). Interaction Design: Beyond HumanComputer Interaction, New York: John Wiley.

SÁ, M. AND E. CHURCHILl (2013). Mobile Augmented Reality: A Design Perspective. Human Factors in Augmented Reality Environments. W. Huang, L. Alem and M. A. Livingston, Springer New York: 139-164.

SATO, M., ET AL. (2007). Building a customizable user interface framework using hyperlinks for smart devices. Proceedings of the 5th IFIP WG 10.2 international conference on Software technologies for embedded and ubiquitous systems. Santorini Island, Greece, Springer-Verlag.

SAVio, N. \& BRAiterman, J. (2007). Design Sketch: The Context of Mobile Interaction. Mobile HCI 2007. Singapore.

SCHILIT, B. N., ET AL. (2002). "Web interaction using very small Internet devices." Computer 35(10): 37-45.

TUllis, T. \& ALBERT, в. (2008). Measuring the User Experience, San Francisco, Morgan Kaufmann.

\section{Sobre a autora}

\section{Heloisa Candello}

$<>$

$\mathrm{PhD}$ em Tecnologias Interativas (University of Brighton - UK); Mestre em Multimedia (UNICAMP - Brazil), graduação em Design na UFSC. Pesquisa sobre interação humano-computador.

Artigo recebido em 10 ago. 2013, aprovado em 14 dez. 2013. 